grossen Ueberschuss von Oel. Leinöl vermag diese Reaktionsprodukte zu einem höheren Prozentsatze aufzulösen als Holzöl, ohne dabei zu erstarren.

W. Fahrion ${ }^{1}$ ) wendet sich ebenfalls gegen die Auffassung der Holzölgelatine als einheitliches Produkt einer mesomorphen Reaktion, die Schapringer in einer Doktordissersation im Kronsteinschen Sinne aufrecht zu erhalten bestrebt ist. Ueber die Verwendung des Holzöles zu Konservenlacken ${ }^{2}$ ) wurde in der Farbenzeitung diskutiert.

Ueber den Wert der Gelatinierungstemperatur zur Untersuchung von chinesischem Holzöl berichten W. Höpfner und Burmeister ${ }^{3}$ ).

Ueber Klebstoffe und Bindemittel referiert $\mathrm{Kausch}{ }^{4}$ ).

\section{Patente.}

Karl Louis Felix Friedemann ${ }^{5}$ ) ist ein Verfahren geschützt zur Auflösung der Leinöl-

1) Farben-Ztg. Jahrg. 18, pp. 2418 ff.

F) Farben-Ztg. Jahrg. 18, p. 1117.

8) Chem.-Ztg. 37, pp. 18/19.

4) Kunststoffe 3, p. 63 ff. und p. 89 ff.

5) D. R. P. $258853, \mathrm{Kl} .22 \mathrm{~h}$, vom 20.9 . datiert. oxydationsprodukte durch Fettsäurelösungen, die bei mässiger oder niederer Temperatur siedend angewendet werden. Z. B. wird die Lösung in Essigsäure gelöst und die Essigsäure nachher verdampft. Die Lösungen sollen zur Imprägnierung von Textilware dienen. $\mathrm{Ob}$ dies Verfahren besser ist als die bisher geübten, erscheint dem Referenten zweifelhaft.

Der Firma S. H. Cohn ${ }^{x}$ sind Verfahren geschützt worden zur Herstellung von glänzend trockenen Lacken aus Holzöl ohne die Verwendung anderer fetter Oele oder Harze, dadurch gekennzeichnet, dass man Holzöl mit Terpenen oder deren Abkömmlingen vom Mindestsiedepunkt $170^{\circ}$ versetzt. Im Zusatzpatent werden auch ranzige Terpentinöle und Kienöle geschützt.

H. Kämpfe ${ }^{2}$ ) stellt aus Tranen Firnisse dar durch fraktionierte Destillation mit gespanntem Wasserdampf.

(Schluss folgt.)

1) D. R. P. 257601 , Kl. $22 \mathrm{~h}$, vom 7. 2. datiert und Zusatzpatent $22431, \mathrm{Kl}, 22 \mathrm{~h}$, vom 5. 10.1912 datiert.

2) D. R, P. 46572, Kl. $22 \mathrm{~h}$, vom 16. 12.1912 datiert.

\title{
Die unverseifbaren Bestandteile natürlicher und gehärteter Fette.")
}

Von Professor J. Marc us on und Dr. G. Me yerheim.

(Mitteilung aus dem Königlichen Materialprüfungsamt za Berlin-Lichterfelde W.)

Den Ausgangspunkt der vorliegenden Untersuchung bildete die Prüfung einer unter der Bezeichnung -Rindertalg، eingereiehten Probe, welche ungewöhnlich hohe Jodzahl $(64,8)$ zeigte. Alsbald trat der Verdacht auf, dass nicht Rindertalg, sondern ein dem Talgol ăhnliches gehärtetes Fett vorläge. Derartige Produkte sollen sich nach $\mathbf{A}$. W. K n a p p ) durch einen geringen Gehalt an Nickel (Reste des zum Hydrieren verwendeten Kataly. eators) kennzeichnen lassen. Im vorliegenden Falle war Nickel durch Ausziehen des Fettes mit heisser Salzsäure und Prüfung des Verdampfungsrückstandes mit alkoholischem Dimethylglyoxim nicht scharf nachweisbar. Es ist aber daranf hinzuweisen, dass nach Angaben von $B$ ö $\mathrm{m} \mathrm{e} \mathrm{r}^{2}$ ) mit Nickel gehärtete Fette nur dann nachweisbare Mengen des Metalles enthalten, wenn die zur Hydrierung verwendeten Oele merkliche Mengen freier Fettsäuren zeigten. Ferner ist zn berücksichtigen, dass ausser Nickel auch Platin, Palladium usw, als Katalysatoren Verwendung finden. Zur Kennzeichnung von Talgol und anderen aus Tran erzeugten Fetten ist zwar in der Literatur noch eine Reihe von Farbenreaktionen angegeben "). Da aber Farbenreaktionen unsicher sind und leicht zu Tänschung Anlass geben können, schien es geboten, die Beurteilung des eingesandten Fettes auf zuverlässigere Grundlagen zu stellen. Durch Behandlung des Fettes mit alkoholischer Digitoninlösung und Spaltung des gebildeten Digitonids nach dem unlängst von $J$. Ma r c a s s o n und $H$. S c h i l li n g veröffentlichten Verfahren ${ }^{4}$ ) konnte reines Cholesterin abgeschieden werden. Nach diesem Befunde konnte die hohe Jodzahl der Probe nicht durch Gegenwart von Pflanzenfetten bedingt sein, sie musste vielmehr auf tierische, dem Rindertalg fremde Fette zurückgeführt werden. Letztere konnten sowohl von Landtieren wie von Seetieren herrühren. Um hierüber Klarheit za gewinnen, wurde die invere Jodzahl des Fettes bestimmt; sie ergab sich zu 111. Hiernach war auf Gegenwart yon Seetierfett zu schliessen, da die innere Jodzabl der Fette und Oele von Landtieren 100 nicht übersteigt; bei einem Rindstalg z. B. zu 89 bestimmt wurde. Die Bestimmung der inneren Jodzahl einer zum Vergleich herangezogenen Probe von Talgol ergab den Wert 107, welcher mit dem des nntersuchten Fettes nahe übereinstimmt. Anch die uibrigen Konstanten des Fettes lagen in derselben Höhe wie die des Talgols. Insbesondere war charakteristisch, dass aus den nnverseifbaren Anteilen beider Materialien ein fester, gesättigter Alkohol der Fettreihe (Octodecylalkohol) vom F. 59-60 abgeschieden werden konnte, der anch in dem Waltran Nr. 2 der Tabelle 1 aufgefunden wurde. Aus der Höhe der für Talgol gefandenen inneren Jodzahl (107) ist bezüglich des Bydrierungsverlaufes noch zu schliessen, dass die Härtung nicht ausgesprochen stufenweise derart verlänft, dass die am stärksten ungesättigten Säuren zunächst vollständig reduziert werden; vielmehr dürfte ein Teil der stark ungesättigten Säuren auch dann noch unhydriert bleiben, wenn bereits die am wenigsten ungesättigte Oelsäure zum grossen Teil in Stearinsäure übergeführt ist.

*) Mit Erlaubnis der Redaktion und der Verfasser der , Zeltschrift für angewandte Chemie ', Jahrg, 27, Nr. 28 entnommen. 1) Analyst 38, 102 (1913); Chem. Zentralbl. 1913, I, 1471.

2) Z. Unters. Nahr.- a. Genussm. 24, 109, (1912).

3, G r i m me, Chem. Revue $\mathbf{2 0}, 129$ (1913).

) Chem.-Ztg. 37, 1001 (1913). 
Tabelle 1.

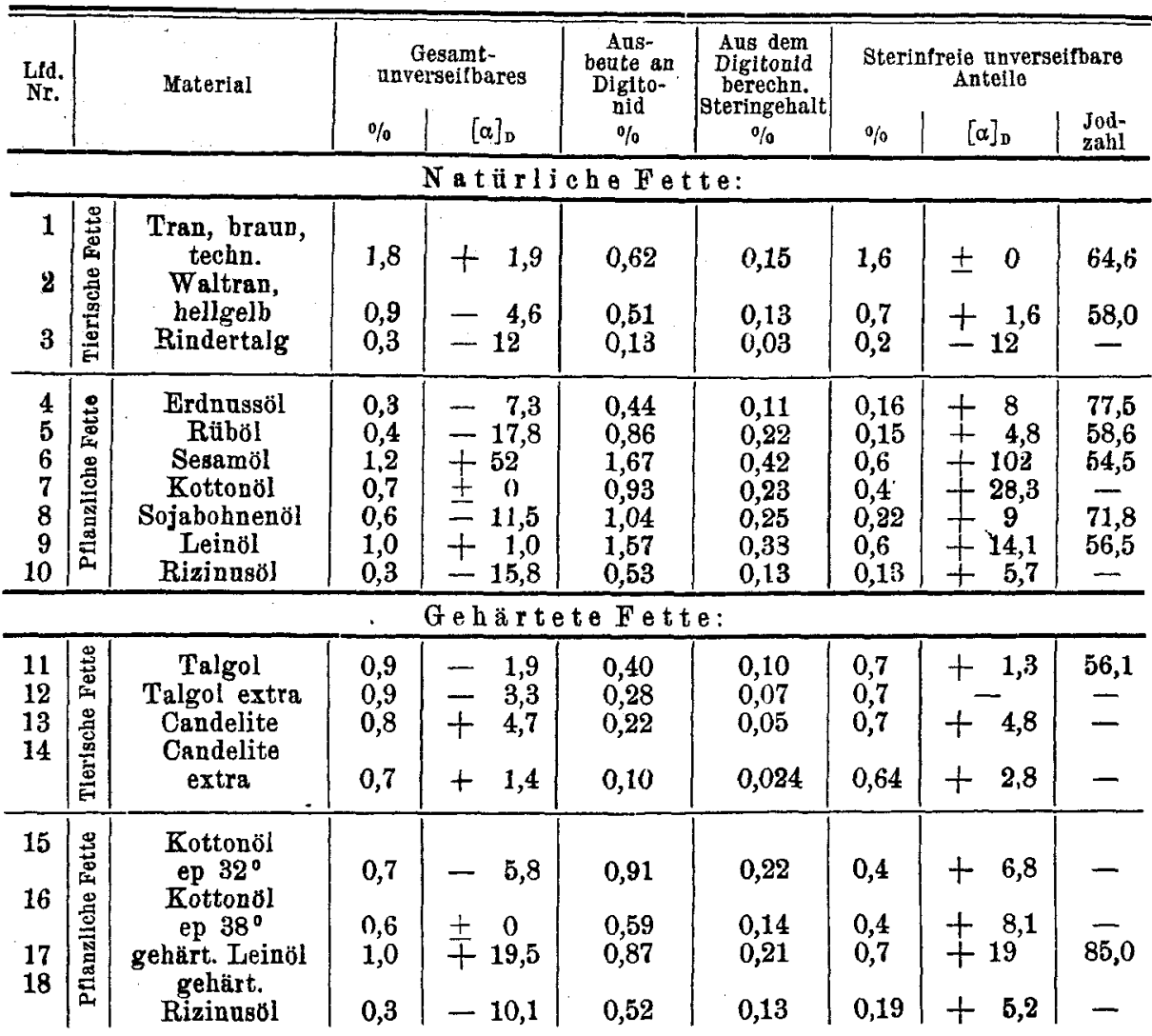

Bei Ausführung der Digitoninprobe war beobachtet worden, dass die Menge des erhaltenen Cholesterinacetates (ebenso wie bei der Prüfung des Talgols) wesentlich geringer war als bei allen bis dahin untersuchten natürlichon Fetten. Hiernach lag die Annahme nahe, dass beim Härten der Fette auch ein Teil des Cholesterins bzw. Phytostering hydriert wird, was nach den Untersuchungen von $\mathrm{B}$ o $\mathrm{me} \mathrm{r}^{1}$ ) nicht za erwarten war. Zar Klärung der Sachlage erschien es geboten, an einer Reihe natürlicher nnd gehärteter Fette den Gehalt an Sterinen quantitatip $z a$ ermitteln. Zwar liegt in der Literatur schon eine grosse Anzahl von Bestimmungen vor, diese beziehen sich aber zumeist nur auf Rohcholesterin, d. h. Gesamtunverseifbares. Quantitative Ermittlung der reinen Sterine ist zurzeit nur durch Fällen der unverseifbaren Anteile mit Digitonin möglich. Zur Ausführung entsprechender Untersuchungen wurden von natürlichen Fetten Talg, Tran, Rüböl, Erdnussöl, Kottonöl, Sesambl, Sojabohnenöl, Leinol und Rizinusöl, von gehärteten Fetten Talgol, Talgol extra, Candelite, Candelite extra, gehärtetes Kottonठl, Leinöl und Rizinusöl verwandt. Die Abscheidnng des Gesamtunverseifbaren erfolgte teils nach $B$ o $m$ e $\left.r^{2}\right)$, teils durch Herstelinug von Kalisalzen der Fettsäuren, Verreiben der getrockneten Produkte mit Sand, Ausziehen mit Aether and nochmalige Verseifung des Extraktes. Bei letzterer Arbeitsweise ist der Verbrauch an Aether geringer, die Versnchsansführung danert jedoch länger; die Ausbeuten sind bei beiden Verfahren naheza die gleichen. Behnf Ansfällnng der Sterine wurden die unverseifbaren Anteile in warmem Alkohol gelöst und mit $50 \mathrm{ccm}$ $1 \%$ iger alkoholischer Digitoninlossung versetzt. Der Niederschlag wurde nach zwölfstündigem Stehen abgesaugt, das Filtrat etwas eingeengt and von neuem mit Digitoninlösung versetzt; diese Operation wurde wiederholt, bis kein Niederschlag mehr auftrat. Die gesammelten Digitonide wurden bei $105^{\circ}$ getrocknet und gewogen. Ans der Menge des Digitonids wurde der Gehalt an Sterinen nach $W$ in d a a s') durch Multiplikation mit 0,2431 berechnet. Die erhaltenen Ergebnisse sind in Tabelle 1 zusammengestellt.

\section{Unrerselfbare Bestandteile natürlicher Fette.}

Wio ans Tabelle 1 hervorgeht, schwankt der Steringehalt der untersuchten natürlichen Fette von 0,03 bis $0,38 \%$; den hochsten Wert ergab Luinöl, den niedrigsten Rindertalg ${ }^{4}$ ). Weiterhin ist ersichtlich, dass die Sterine nicht, wie bisher vielfach angenommen wurde, den Hauptbestandteil des Gesamtunverseifbaren ausmachen; der Prozentgehalt des Unverseifbaren an Sterinen schwankte bei den untersuchten pflanzlichen Oelen von 33-55\%,

1) Z. Unters. Nahr.- a. Genussm. 24, 108, (1912)

2) Unters. Nahr.- a. Genussm. 1898, 21 ; Ma re us o n, Laboratoriumsbuch für die Industrie der Oele und Fette $1911,61$.

Z. physiol. Chem. 65, 110 (1910).

4) Quantitative Bestimmung des Steringehaltes natürlicher Fette mittels Digitonin hat auch K10sterman (Z. Uuters. Nahr.- u. Genussm. 26, 483 [1913]) in Aussicht genommen; beim Erscheinen seiner Notiz waren aber die vorstehenden Bestimmungen schon im wesentlichen abgeschlossen. 
bei den tierischen Oelen (Tran und Talg) von 8-14\%. Mit dem Gehalt der unverseifbaren Anteile an Sterin steht in einem gewissen Zusammenhange ihr optisches Drehungsvermögen. Die Sterine sind stark linksdrehend, und zwar dreht nach Literaturangaben ${ }^{1}$ ) Cholesterin $[\alpha]_{\mathfrak{v}}=-31,1^{\circ}$ in Aetherlösung, Phytosterin $[\alpha]_{\mathrm{v}}=-34,2^{\circ}$. Dementsprechend sollte das Gesamtunverseifbare nm so stärkere Linksdrehung aufweisen, je höher der Steringehalt ist. Tatsächlich kommen aber, wie weiter unten gezeigt werden wird, in dem Unverseifbaren neben den linksdrehenden Sterinen noch rechtsdrehende, zumeist unbekannte Alkohole vor, welche die Linksdrehung der Sterine zum Teil anfheben (wie beim Kottonöl), teilweise sogar Rechtsdrehuvg des Gesamtunverseifbaren bedingen (Leinöl, Tran und Sesamøi). Besonders auffallend ist die starke, durch Gegenwart von Sesamin bedingte Rechtsdrehung des Sesamölunverseifbaren $\left([a]_{\mathrm{D}}=+52^{\circ}\right)$. Dieses Verhalten wird in Fällen, in denen Farbenreaktionen versagen, zum Nachweis des Sesamöles verwendet werden können.

Die von den Sterinen und überschïssigem Digitonin befreiten unverseifbaren Anteile zeigten mejst dickölige bis salbenartige Beschaffenheit. Sie bestanden im wesentlichen aus ungesättigten, rechtsdrehenden, nur im Falle des Rindertalges linksdrehenden Alkoholen, daneben warden zum Teil geringfügige Mengen von Kohlenwasserstoffen nachgewiesen. Nur im Unverseifbaren des dunklen Tranes waren beträchtliche Mengen von Kohlenwasserstoffen enthalten. Bemerkenswert ist die ausserordentlich starke Rechtsdrehung der von Sterinen befreiten unverseifbaren Anteile des Sesamöles $\left([a]_{D}=+102 \%\right.$. In letzteren findet offenbar eine starke Anreicherung des rechtsdrehenden Sesamins statt, das durch Digitonin nicht gefällt wird.

Die Jodzahl der sterinfreien Anteile schwankte bei den untersuchten Fetten von 56-78 (nach H ü bl.

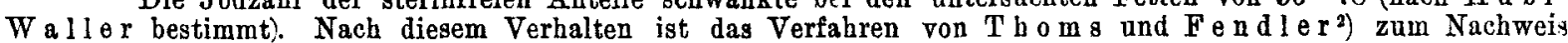
geringer Mengen Mineralöl in Leinöl, das auf der Bestimmung der Jodzahl des Gesamtunverseifbaren beruht, in gleicher Weise für die übrigen fetten Oele verwendbar. Da nämlich die von Sterinen befreiten Anteile eine Jod. zahl von 56-78 zeigen, muss auch das sterinhaltige Gesamtunverseifbare nahezu das gleiche Jodabsorptions. vermögen aufweisen (Jodzahl der reinen Sterine etwa 68). (Schluss folgt.)

1) Belis tein Bd. II, 1071, 1075

9) Chem.-Ztg. 28, 841 (1904).

An die Redaktion

\section{Korrespondenz,}

\section{der „Chemischen Revue über die Fett- und Harz-Industrie““}

$\mathrm{Hamburg}$.

Im Bulletin der Section Scientifique de l'Académie Roumaine von 1913, Band 2, Seite 84, wurde meine Abhandlung über die Bestimmung der aromatischen und ungesättigten Kohlenwasserstoffe (Carbüren) im Erdöl und seinen Produkten veröffentlicht. Das Prinzip der dort angeführten Methode beruht auf dem von mir in die Grossindustrie eingeführten Verfahren der Raffination von Petroleumprodukten mittels schwefliger Säure. Diese Abhandlung wurde der rumänischen Akademie der Wissenschaften von Prof. Mrazec in $\mathrm{m}$ e in e $\mathrm{m}$ Namen überreicht. Die Ueberschrift des Aufsatzes trägt ausdrücklich an erster Stelle meinen Namen.

In Heft 3, Jahrgang 21, Seite 64 ihrer Zeitschrift wurde ein aus dem Chemisch-Technischen Repertorium entnommenes Referat veröffentlicht, in welchem irrtümlicherweise Herr Mrazec als Verfasser der Abhandlung genannt wird.

$\mathrm{Da}$ infolge dieses Irrtums mir leicht eine moralische und materielle Schädigung erwachsen könnte, wäre ich Ihnen sehr verbunden, wenn Sie in der nächsten Nummer der »Chemischen Revue darauf hinweisen würden, dass im Chemisch-Technischen Repertorium irrtümlicherweise Herr Mrazec als Verfasser angegeben war und infolgedessen dieser Irrtum auch in Thre Zeitschrift übernommen worden ist.

Mit verbindlichstem Dank im voraus zeichne ich

Berlin W. 8, den 5. März 1914.

mit vorzüglicher Hochachtung

Dr. L. Edeleanu.

\section{Zur Untersuchung von Asphalten.}

Ueber meine unter diesem Titel in der Chemiker-Zeitung 1914, Nr. 2, p. 18, erschienene Arbeit referierte $\mathrm{K}$. in dieser Zeitschrift in $\mathrm{Nr}$. 3 dieses Jahrganges und er gab seinem kurzen Referat einige persönliche Bemerkungen bei.

Sowohl das Referat als auch die persönliche Notiz entsprechen nicht meinen Ausführungen. Es mag hier kurz daran erinnert werden, dass ich bereits früher ${ }^{1}$ ) denselben Gegenstand ausführlich behandelte und meine letzte Veröffentlịchung, über die $\mathrm{K}$. referierte, beschäftigt sich nur mit

1) Chem. Ztg. 1912, pp. 4 und 22. 